\title{
A realistic generic model for anti-tetrachiral systems
}

\author{
Ruben Gatt ${ }^{*, 1}$, Daphne Attard ${ }^{1}$, Pierre-Sandre Farrugia ${ }^{2}$, Keith M. Azzopardi', \\ Luke Mizzi', Jean-Pierre Brincat', and Joseph N. Grima ${ }^{1,3}$ \\ ${ }^{1}$ Metamaterials Unit, Faculty of Science, University of Malta, Msida, MSD 2080, Malta \\ ${ }^{2}$ Department of Physics, Faculty of Science, University of Malta, Msida, MSD 2080, Malta \\ ${ }^{3}$ Department of Chemistry, Faculty of Science, University of Malta, Msida, MSD 2080, Malta
}

Received 22 June 2013, revised 25 July 2013, accepted 12 August 2013

Published online 16 September 2013

Keywords analytical modeling, finite element analysis, negative Poisson's ratio, Young's modulus

*Corresponding author: e-mail ruben.gatt@um.edu.mt, Phone: +356 23402274

Chiral systems are a class of structures, which may exhibit the anomalous property of a negative Poisson's ratio. Proposed by Wojciechowski and implemented later by Lakes, these structures have aroused interest due to their remarkable mechanical properties and numerous potential applications. In view of this, this paper investigates the on-axis mechanical properties of the general forms of the flexing anti-tetrachiral system through analytical and finite element models. The results suggest that these are highly dependent on the geometry (the ratio of ligament lengths, thicknesses, and radius of nodes) and material properties of the constituent materials. We also show that the rigidity of an anti-tetrachiral system can be changed without altering the Poisson's ratio.

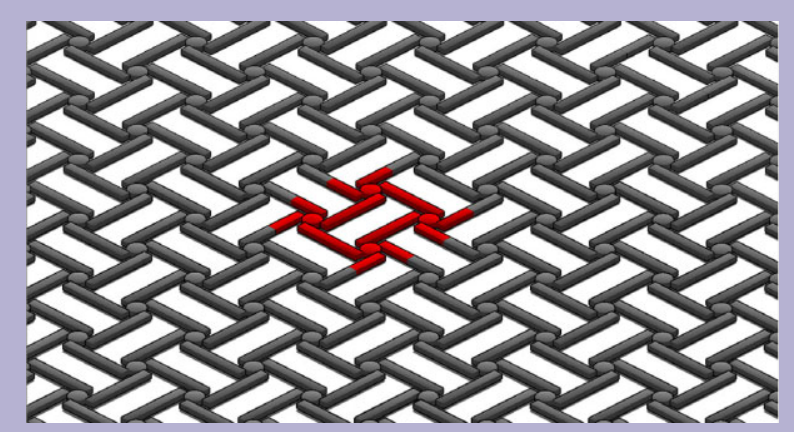

The anti-tetrachiral system, with the unit cell shown in red.
1 Introduction Auxetic materials, i.e. materials, which expand laterally when a uniaxial load is applied, have received a significant amount of interest in the past few decades [1-40]. Auxetic character is known to be a scale independent characteristic [3, 4], being the product of particular geometries undergoing a specific deformation mechanism. This means that auxetic behavior can be found operating at the macro[4-30] micro- [4, 31-33] or nano-scales [34-42].

One particular mechanism that has attracted a lot of attention in recent years is that of chiral systems. These systems were first proposed by Wojciechowski [7], and later implemented as a structure by Lakes [9, 10]. Lakes' hexagonal chiral honeycomb is comprised of a central cylinder (referred to as "node") with six tangentially attached ligaments, exhibiting a rotational symmetry of order six. Prall and Lakes [10] determined that this network was isotropic with an in-plane Poisson's ratio of -1 . Later, Sigmund et al. [11] proposed a 3D system which, in one plane, has a unit-cell made up of nodes with four attached ligaments, with each cell containing an equal amount of right and left handed units. This system was dubbed as an "antitetrachiral" honeycomb system by Grima $[12,13]$. These are only two examples of a larger set of structures, which include the tetrachiral, trichiral, and anti-trichiral systems $[12,13]$. Furthermore, if the rotational symmetry constrain is relaxed, another class of structures called the meta-chiral systems [12] can be produced.

Chiral systems have piqued the interest of several researchers due to their numerous potential applications, which include satellite antennas designs [14, 15], sensors [16], and stent geometries [17]. This has resulted in their mechanical properties being extensively studied by several researchers [7, 9-28].

This paper investigates the general forms of the antitetrachiral system (henceforth referred to as general antitetrachirals) where the ligaments are glued to the external 


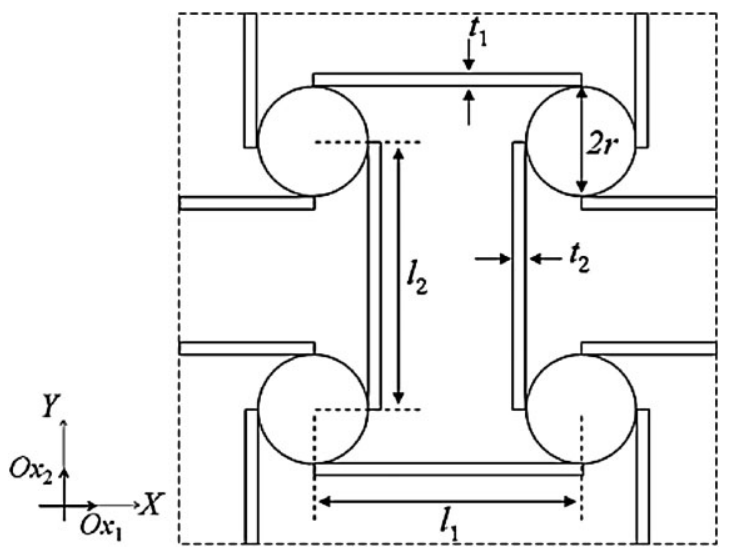

Figure 1 The general anti-tetrachiral system. The unit cell is highlighted by a dashed box.

surface of the nodes, as illustrated in Fig. 1. In particular, analytical models and finite element analysis (FEA) were used to study systems in which the ligaments aligned in the $O x_{1}$ directions may have different geometric and mechanical properties than those aligned in the $O x_{2}$ direction.

2 Analytical modeling of the general antitetrachiral systems with circular nodes In order to understand more clearly the properties of the general flexing anti-tetrachiral, an analytical model, which predicts the Poisson's ratio and Young's moduli in terms of the different lengths, thicknesses, and stiffness of the ligaments was derived. For this model, the unit cell shown in Fig. 1 was considered, where the nodes have a radius $r$ and are made of a material having Young's modulus $E_{\mathrm{s}}$. The ligaments in the $O x_{1}$ direction have a length $l_{1}$, a thickness of $t_{1}$ and are made of a material having a Young's modulus of $E_{\mathrm{s} 1}$. Similarly, the ligaments in the $O x_{2}$ direction have a length $l_{2}$, a thickness of $t_{2}$ and are made of a material having a Young's modulus of $E_{\mathrm{s} 2}$.

In the un-deformed conformation, the projections in the $O x_{i}$ directions (where $i=1,2,3$ ) are given by:

$$
X_{1}=2 l_{1}, \quad X_{2}=2 l_{2}, \quad X_{3}=z
$$

where $z$ is the thickness in the third direction of the nodes and ligaments.

In the derivation, it was assumed that all deformations occur as a result of flexure of the ligaments. In particular:

- the lengths of the ligaments remain constant (i.e. the ligaments do not stretch)

- the nodes remain perfectly rigid and simply rotate by an angle $\phi$ which is equal to the angular deflection at the ends of the ligaments

- the line joining the center of the nodes with the point of contact between the ligaments and the nodes remain at $90^{\circ}$ to the ligaments (i.e. the point of contact between the ligaments and nodes is not acting as a hinge).
In other words, the analytical model only captures deformations, which occur as a result of "flexure" of the ligament.

2.1 The in-plane Poisson's ratio for loading on-axis The Poisson's ratio $\left(v_{i j}\right)$ in the $O x_{1}-O x_{2}$ plane for loading in the $O x_{i}$ may be defined as:

$$
v_{i j}=-\frac{\varepsilon_{j}}{\varepsilon_{i}}
$$

where $\varepsilon_{i}$ and $\varepsilon_{j}$ are the engineering strains in the $O x_{i}$ direction $(i=1,2)$. These strains may be expressed as:

$$
\varepsilon_{1}=\frac{\Delta X_{1}}{X_{1}^{\text {init }}} \quad \text { and } \quad \varepsilon_{2}=\frac{\Delta X_{2}}{X_{2}^{\text {init }}}
$$

where $X_{1}^{\text {init }}$ are the projections of the un-deformed unit cell in the $O x_{i}$ directions and $\Delta X_{i}$ are small changes in length in $X_{1}^{\text {init. }}$

If it is assumed that the on-axis uniaxial loads $\sigma_{i}$ in the $O x_{i}$ direction result in flexure of the ligaments which will cause a rotation of the circular nodes by the angle $\phi$, as shown in Fig. 2, then the distance between the centers of the nodes will change from $l_{\mathrm{i}}$ to $\left(l_{\mathrm{i}}+2 e_{i}\right)$ where $e_{i}$ is equal to:

$$
e_{i}=2\left(r+\frac{t_{i}}{2}\right) \sin (\phi) \approx 2\left(r+\frac{t_{i}}{2}\right) \phi .
$$

Thus, the strains in the $O x_{i}$ directions are given by:

$$
\varepsilon_{i}=\frac{\Delta X_{i}}{X_{i}^{\text {init }}}=\frac{2(2 e)}{2\left(l_{i}\right)}=\frac{2\left(r+\frac{t_{i}}{2}\right) \phi}{l_{i}}=\frac{\left(2 r+t_{i}\right) \phi}{l_{i}}
$$

and hence the Poisson's ratio is given by:

$$
\begin{aligned}
v_{i j} & =-\frac{\varepsilon_{j}}{\varepsilon_{i}}=-\frac{\left(2 r+t_{j}\right) \phi}{l_{j}} \cdot \frac{l_{i}}{\left(2 r+t_{i}\right) \phi} \\
& =-\frac{\left(2 r+t_{j}\right) l_{i}}{\left(2 r+t_{i}\right) l_{j}} .
\end{aligned}
$$

2.2 The in-plane Young's modulus for loading on-axis The Young's modulus in the $O x_{i}$ direction may be

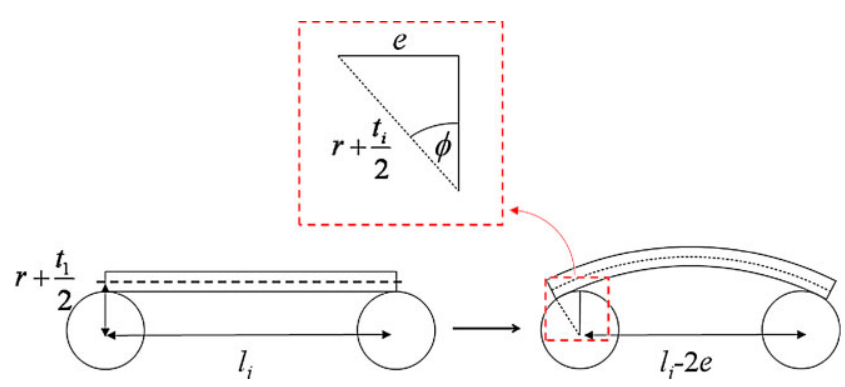

Figure 2 The changes that take place when a ligament undergoes flexing. 
derived through the conservation of energy approach, where it must be emphasized that:

- since a pure "flexure" model is being assumed, all the strain energy will be stored as "bending energy" in the ligament. (This means that the length to thickness ratio if the ligaments must have a lower limit of 10.)

- the energy stored in the ligaments lying in the $O x_{1}$ direction will be different from the energy stored in ligaments lying in the $O x_{2}$ direction if these ligaments have different lengths, thickness, and/or modulus.

An applied strain $\varepsilon_{i}$ in the loading direction may be related to the strain energy per unit volume $U$ through:

$$
U=\frac{1}{2} E_{i} \varepsilon_{i}^{2}
$$

where $E_{i}$ is the Young's modulus of the structure. This strain energy per unit volume may be related to the work done per unit cell $W_{\mathrm{T}}$ through:

$$
U=\frac{W_{\mathrm{T}}}{V_{\text {Cell }}}
$$

where $V_{\text {Cell }}$ is the volume of the unit cell given by:

$$
V_{\text {Cell }}=X_{1} X_{2} X_{3}=4 l_{1} l_{2} z
$$

and $W_{\mathrm{T}}$ is the total work done by a single unit cell, which is equal to the bending energy stored in the deformed ligaments inside the unit cell. As shown in Fig. 1, each unit cell contains eight ligaments, four of which are aligned with the $O x_{1}$ direction whilst the other four are aligned with the $O x_{2}$ direction, i.e.:

$$
W_{\mathrm{T}}=4\left(W_{\mathrm{Lig} 1}+W_{\mathrm{Lig} 2}\right)
$$

where $W_{i}$ is the bending energy in the ligaments aligned in the $O x_{i}$ direction $(i=1,2)$, and as highlighted above $W_{1}$ is in general not equal to $W_{2}$. Nevertheless, although these ligaments may have different geometric parameters and different mechanical properties, the angle by which they flex, measured at the point of contact with the node, is geometrically constrained to be equal to the angle $\phi$ by which the nodes would have rotated. This angle can be related to the bending moment $M_{\varphi}$ through standard beam theory [43]:

$$
\phi=\frac{M_{\phi i} l_{i}}{2 E_{\mathrm{s} i} I_{i}}
$$

where $E_{\mathrm{s} i}$ is the intrinsic Young's modulus of the material of the ligament $i$ whilst $I_{i}$ is the second moment of area of the ligament $i$ in the $O x_{i}$ direction, which is given as:

$$
I_{i}=\frac{t_{i}^{3} z}{12} .
$$

The energy $W_{i}$ stored in each flexed ligament $i$ may be obtained from:

$$
W_{i}=2 \int_{0}^{\phi} M_{\phi i} \mathrm{~d} \phi
$$

i.e. from Eqs. (11) and (13), the energy $W_{i}$ is given by:

$$
\begin{aligned}
W_{i} & =2 \int_{0}^{\phi} M_{\phi i} \mathrm{~d} \phi=2 \int_{0}^{\phi}\left(\frac{z E_{\mathrm{si}} t_{i}^{3}}{6 l_{i}} \phi\right) \mathrm{d} \phi \\
& =\frac{z E_{\mathrm{si}} t_{i}^{3}}{6 l_{i}} \phi^{2} .
\end{aligned}
$$

Thus, from Eqs. (10) and (14), the total energy per unit cell is equal to:

$$
\begin{aligned}
W_{\mathrm{T}} & =4\left(W_{\mathrm{Lig} 1}+W_{\mathrm{Lig} 2}\right) \\
& =4 z\left(\frac{E_{\mathrm{s} 1} t_{1}^{3}}{6 l_{1}}+\frac{E_{\mathrm{s} 2} t_{2}^{3}}{6 l_{2}}\right) \phi^{2}
\end{aligned}
$$

and from Eqs. (5), (7)-(9), and (15), the Young's modulus for loading in the $O x_{i}$ direction $(i=1,2)$ is given by:

$$
E_{i}=\frac{1}{3\left(2 r+t_{i}\right)^{2}} \frac{l_{i}}{l_{j}}\left(\frac{E_{\mathrm{s} 1} t_{1}^{3}}{l_{1}}+\frac{E_{\mathrm{s} 2} t_{2}^{3}}{l_{2}}\right) \quad(i=1,2) .
$$

3 Finite element modeling Finite element (FE) simulations were carried out to examine the validity of the assumptions made during analytical modelling, i.e. (1) the anti-chiral system constructed from ligaments glued to circular nodes deforms primarily through flexure of the ligaments and (2) the on-axis in-plane Poisson's ratios and Young's moduli are approximated by the expressions in Eqs. (6) and (16), respectively.

In line with previous work [23], four dimensional parameters, $\alpha_{1}, \alpha_{2}, \beta_{1}$, and $\beta_{2}$, were defined, where $\alpha_{1}=l_{1} / r$, $\alpha_{2}=l_{2} / r, \beta_{1}=t_{1} / r$, and $\beta_{2}=t_{2} / r$. Given the symmetry of the structure and the loading conditions, the system illustrated in Fig. 1 was constructed in ANSYS (v12.0) [44] running on a supercomputing cluster as a quarter unit cell using the boundary conditions shown in Fig. 3. This produces a valid representation of an infinite system.

The structure was aligned in such a way that the $O x_{1}$ axis in the analytical model was parallel to the $X$-direction in ANSYS, to be able to compare the two models directly. Various sets of simulations were carried out which were based on systems having different geometric parameters and intrinsic mechanical properties, with two parameters being varied simultaneously in a series of sweeps. In the first three sweeps, the first chosen parameter was varied continuously, while the second chosen parameter was fixed at one of three defined values, until all possible combinations were exhausted. In the second three sweeps, the first chosen variable was fixed at one of three set values, while the second chosen parameter was varied continuously, until all possible combinations were exhausted. The complete set of 


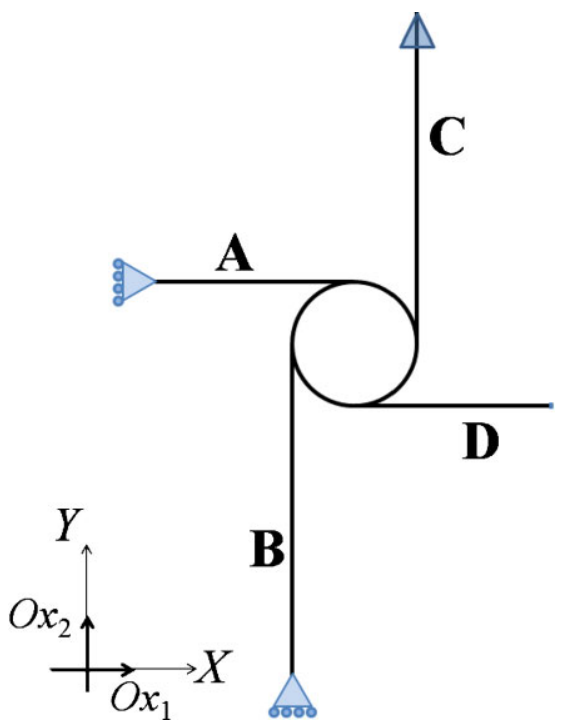

Figure 3 The boundary conditions which were applied to the model constructed in ANSYS to represent an infinite system. The end of ligament A was constrained not to move in the $O x_{1}$ direction, the end of ligament $\mathrm{B}$ was constrained not to move in the $\mathrm{Ox}_{2}$ direction, the end of ligament $\mathrm{D}$ was not allowed to rotate whilst a strain of $2 \%$ was applied on the end of ligament $\mathrm{C}$.

parameters and properties varied is listed in the Supporting Information. A representative sample of the parameters used from all the simulations performed is given in Table 1. All systems, including both the nodes and ligaments, were meshed using plane elements (Plane 82, a 2D 8-Node Structural Solid) with plain strain capability in accordance with earlier work [45]. A convergence test was performed to choose an appropriate element size for the systems modeled. In this case, the mesh density was calculated using the ANSYS Smart Size Feature, with the size level set to 1. All the systems were solved linearly. Note that in all simulations, the models were subjected to strains in the $O x_{2}$ direction. Typical values for PVC plastics were used for the constituting materials of the ligaments and nodes [46].

4 Results and discussion A qualitative analysis of the deformed structures as simulated through the FE

Table 1 Three representative sets of FEA simulations that were carried out. The complete list of simulations performed is given in the Supporting Information.

\begin{tabular}{|c|c|c|c|}
\hline \multirow[t]{2}{*}{ set } & 1 & 4 & 10 \\
\hline & $\alpha_{1}, \alpha_{2}$ & $\alpha_{1}, E_{\mathrm{s} 1}$ & $\beta_{1}, \beta_{2}$ \\
\hline$\alpha_{1}$ & $5,6, \ldots, 15$ & $5,6, \ldots, 15$ & 10 \\
\hline$\alpha_{2}$ & $5,10,15$ & 10 & 10 \\
\hline$\beta_{1}$ & 0.1 & 0.1 & $0.05,0.06, \ldots, 0.15$ \\
\hline$\beta_{2}$ & 0.1 & 0.1 & $0.05,0.1,0.15$ \\
\hline$E_{\mathrm{s} 1}(\mathrm{GPa})$ & 3 & $2,3,4$ & 3 \\
\hline$E_{\mathrm{s} 2}(\mathrm{GPa})$ & 3 & 3 & 3 \\
\hline$E_{\mathrm{s} n}(\mathrm{GPa})$ & 4 & 4 & 4 \\
\hline
\end{tabular}

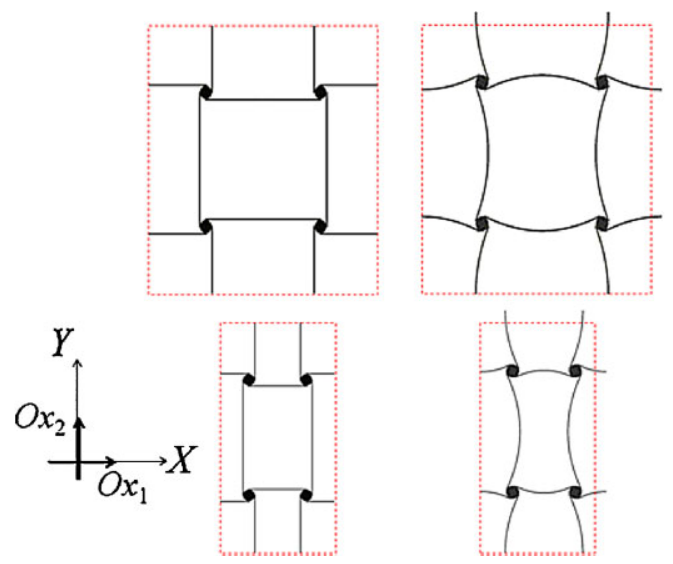

(a)

Figure 4 A representative sample of the general anti-tetrachiral systems simulated in ANSYS where the parameters were changed as follows: (a) $\alpha_{i}=20$ and $\beta_{i}=0.20$, (b) $\alpha_{2}=20$. The other parameters are as follows for both examples: $\alpha_{i}=10, \beta_{i}=0.10$ and $E_{\mathrm{s} i}=3.00 \mathrm{GPa}$ (where $\left.i=1,2\right)$.

simulations suggests that the general anti-tetrachiral systems have negative Poisson's ratios and are observed to deform through flexing of the slender ligaments as in the case of their regular counterparts, which is accompanied by rotation of the circular nodes as illustrated by the images of representative samples of the deformed and un-deformed shapes shown in Fig. 4.

This validates the assumption made in the derivation of the analytical model, namely that the main mode of deformation is that of flexure of the ligaments accompanied by a rotation of the nodes. Furthermore, the figures show that when considering anti-tetrachiral systems having different ligament lengths (i.e. $\alpha_{1} \neq \alpha_{2}$ ), for loading in the $O x_{2}$ direction, when $\alpha_{2}>\alpha_{1}, \alpha_{2}$ tends to assume a smaller curvature than $\alpha_{1}$, and vice versa in the case when $\alpha_{1}>\alpha_{2}$. In the case when $\alpha_{1}=\alpha_{2}$, the ligaments are observed to assume the same curvature.

Plots of the Poisson's ratios and Young's moduli as predicted by the analytical model and the FEA simulations for the systems in Table 1 are given in Fig. 5. These plots further confirm that there is excellent agreement between the predictions made by the simple analytical model derived in Section 2 and the detailed FEA simulations performed in Section 3. Moreover, these plots also highlight the relationship between the Poisson's ratios and Young's moduli with the geometric and intrinsic properties of the materials. The complete set of results for all the simulations performed is given in the Supporting information.

The results obtained for $v_{21}$ versus $\alpha_{2} / \alpha_{1}$ (Fig. 5) clearly suggest that for all values of $\alpha_{1}$ and $\alpha_{2}$ used, the Poisson's ratio is always negative, although not equal to -1 when $\alpha_{1} \neq \alpha_{2}$, as predicted for the regular flexing model [13]. In fact, both the analytical model and simulations suggest that for loading in the $\mathrm{Ox}_{2}$ direction, the Poisson's ratio is observed to become more negative as the ligament $l_{2}$, becomes longer than the ligament $l_{1}$, thus large negative 
Set 1
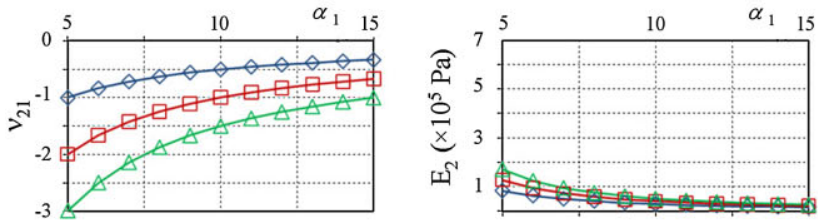

$\diamond \alpha_{2}=5 \square \alpha_{2}=10 \Delta \alpha_{2}=15-\alpha_{2}^{a}=5-\alpha_{2}^{a}=10-\alpha_{2}^{a}=15$

Set 4
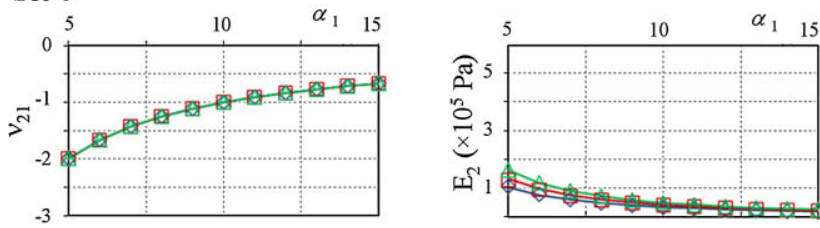

$\diamond E_{S 1}=2 \square E_{S 1}=3 \triangle E_{S 1}=4-E_{S 1}^{a}=2-E_{S 1}^{a}=3-E_{S 1}^{a}=4(\mathrm{GPa})$

Set 10

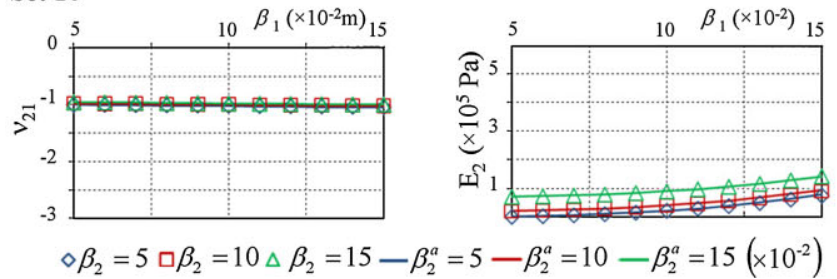

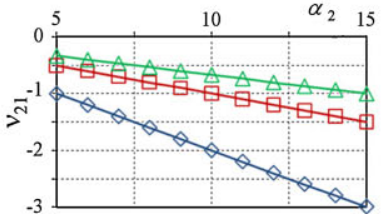

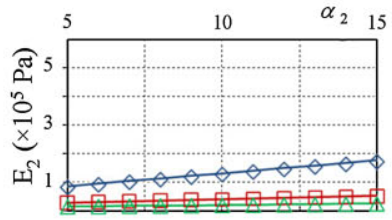

$\diamond \alpha_{1}=5 \square \alpha_{1}=10 \Delta \alpha_{1}=15-\alpha_{1}^{a}=5-\alpha_{1}^{a}=10-\alpha_{1}^{a}=15$
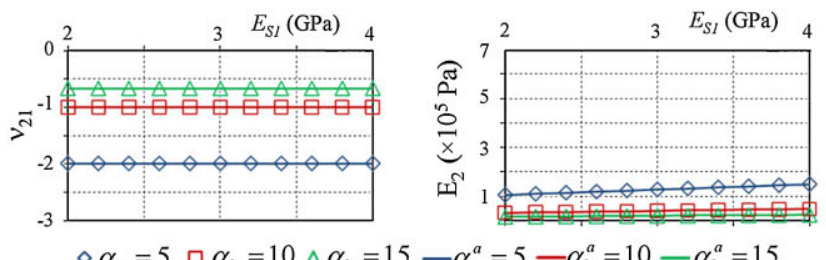

$\diamond \alpha_{1}=5 \square \alpha_{1}=10 \Delta \alpha_{1}=15-\alpha_{1}^{a}=5-\alpha_{1}^{a}=10-\alpha_{1}^{a}=15$
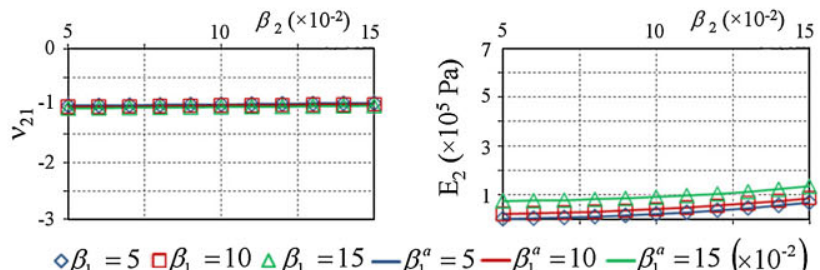

$\diamond \beta_{1}=5 \square \beta_{1}=10 \Delta \beta_{1}=15-\beta_{1}^{a}=5-\beta_{1}^{a}=10-\beta_{1}^{a}=15\left(\times 10^{-2}\right)$

Figure 5 Plots of the Poisson's ratios and Young's moduli as predicted by the analytical model and the FE simulations for the systems in Table 1. Note that in the legends, the letter $a$ stands for analytical.

Poisson's ratios are obtained for large values of the ratio $\alpha_{2} / \alpha_{1}$.

When considering systems having ligaments with different thicknesses (i.e. $\beta_{1} \neq \beta_{2}$ ), referring to Figs. 5 and 6 , the results obtained suggest that the Poisson's ratio is also dependent on the ratio of the thicknesses used, though to a much lesser extent than the ligament lengths. In fact, though for all values of $\beta_{2} / \beta_{1}$ used, the Poisson's ratio $v_{21}$ was always negative, in cases where $\beta_{1} \neq \beta_{2}$, it was not equal to -1 . This is predicted by the regular flexing model [13].

The Poisson's ratio is observed to become more negative as $\beta_{1}$ becomes larger than $\beta_{2}$. This can be explained through

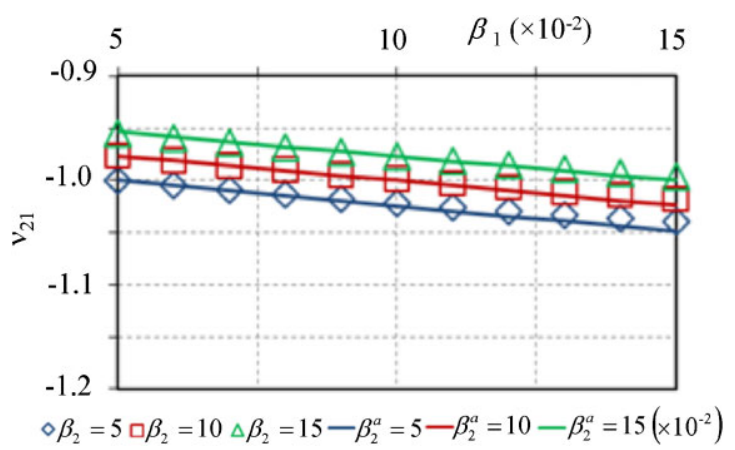

Figure 6 Plots of the Poisson's ratio $\left(v_{21}\right)$ for set $10\left(v_{21}\right.$ vs. $\left.\beta_{1}\right)$ in Fig. 5, where the scale of the Poisson's ratio has been changed in order to show clearly the relation between the plotted variables. the fact that when changing the ratio of the thicknesses, the aspect ratio of the circular node is changed. In other words, referring to Fig. $7 \mathrm{a}$ if $t_{1}=t_{2}$, the distance between the center of the node and the center of the ligament aligned in the $O x_{1}$ (i.e. $r+\left(t_{1} / 2\right)$ ) direction will be the same as the distance between the center of the node and the center of the ligament aligned in the $O x_{2}$ (i.e. $r+\left(t_{2} / 2\right)$ ), hence the node can be described as circular. However, referring to Fig. $7 \mathrm{~b}$, if $t_{1} \neq t_{2}$, the distance between the center of the node and the center of the ligament aligned in the $O x_{1}$ (i.e. $r+\left(t_{1} / 2\right)$ ) direction will not be the same as the distance between the center of the

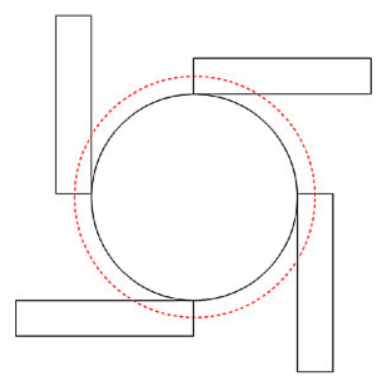

(a)

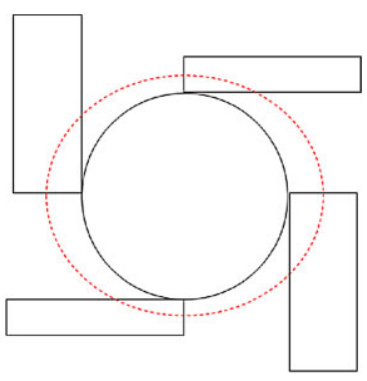

(b)
Figure 7 (a) A node with four ligaments of equal thickness showing that a circle is produced by passing a line from the middle points of these ligaments. (b) A node with four ligaments where the horizontal ligaments have a different thickness from the vertical thickness showing that an ellipse is formed by passing a line from the middle points of these ligaments. 
node and the center of the ligament aligned in the $\mathrm{Ox}_{2}$ (i.e. $r+\left(t_{2} / 2\right)$ ), hence the node is better described as an "elliptical node" rather than as a "circular node". This implies that although the node rotation will be constant, the strains generated in the $O x_{1}$ and $O x_{2}$ directions will be different.

From the results obtained, it may be observed that the Poisson's ratio is independent of the Young's moduli of the constituent materials and radii of the nodes, provided that $\beta_{1}=\beta_{2}$. In fact, for systems where $\alpha_{1}=\alpha_{2}$ and $\beta_{1}=\beta_{2}$ the Poisson's ratio was always constant, with a value of -1 , as predicted for the regular systems. [23] Furthermore, irrespective of the thicknesses of the ligament, in the limit a very small $\beta_{1}$ or $\beta_{2}$, the Poisson's ratio will always tend toward $-l_{i} l_{j}$ (see Eq. 6) Moreover, it must be ensured that the radius of the nodes is small enough compared to the length of the ligaments in order to prevent their overlap, which would otherwise lead to a physically unrealizable structure. This implies that for the simulations performed, the Poisson's ratio is dependent on the ratio of the lengths and thicknesses, where a value of -1 is expected for systems that have equal ligament lengths and thickness, as discussed above.

The Young's moduli of these general anti-tetrachiral systems are dependent on all geometric parameters taken into consideration, as well as the intrinsic Young's moduli of the ligaments. For loading in the $O x_{2}$ direction, the larger the ratio $\alpha_{2} / \alpha_{1}$, the stiffer the structure becomes. This behavior may be explained by considering two systems, each of which has the same $\alpha_{2}$ but has different values for $\alpha_{1}$, such that $\alpha_{1} \neq \alpha_{2}$ as illustrated in Fig. 8a. If these two systems are each given a strain of $10 \%$ in the $O x_{2}$ direction, the nodes in the two structures must each rotate by the same amount to accommodate a flexed vertical ligament that corresponds to $10 \%$ strain. This means that the angular deflections in the short and long horizontal ligaments must be equal to each other, with the result that the strain energy in the short ligament is much larger than that in the longer ligament (short ligaments are more difficult to bend than longer ones) with the result that the Young's modulus $E_{2}$ of the systems with short ligaments is much larger. The same result would be obtained if $\alpha_{1}$ is kept constant and $\alpha_{2}$ is varied. As illustrated in Fig. 8b, the longer the ligament in the direction of loading, the larger the node rotation for the same strain, which means that the ligament in the transverse direction must bend to a higher degree, thus requiring more energy and making the whole structure stiffer in the direction of loading.

The Young's moduli of the general anti-tetrachiral systems are dependent on the ratio between the lengths and node size. In fact, for any particular $\alpha_{2} / \alpha_{1}$ ratio, the systems become stiffer as the radius of the nodes decreases. This can be explained through the fact that for a given strain, the rotation of a large node will be smaller than that of a smaller node, as illustrated in Fig. 8c and indicated by Eq. (5). This will result in less bending of the ligaments for larger nodes so that the amount of energy needed is

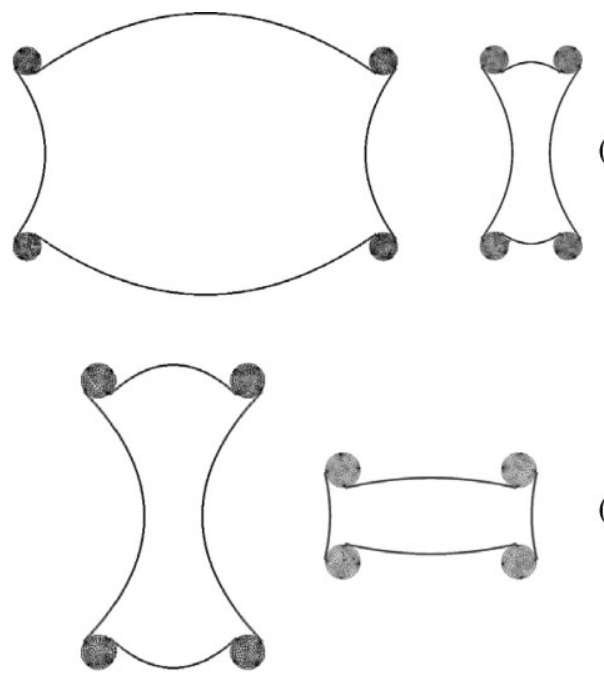

(a)

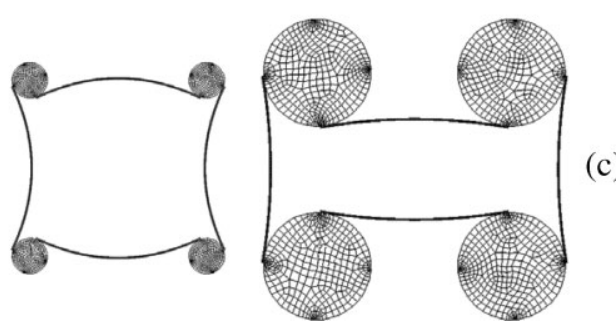

Figure 8 (a) Two anti-tetrachiral systems with $\beta_{2}=15 . \alpha_{1}$ is set at 30 (left) and 10 (right). (b) Two systems with $\alpha_{1}=10 . \alpha_{2}$ is set at 20 (left) and 5 (right). (c) Two systems with identical values for $l_{1}$ and $l_{2}$, but radii, $1 \mathrm{~cm}$ (left) and $3 \mathrm{~cm}$ (right). All of these systems were given a strain of $10 \%$ in the $O x_{2}$ direction.

decreased. Hence, the lower value for the Young's modulus of the anti-tetrachiral.

Furthermore, for loading in the $O x_{2}$ direction, the larger the value of $\beta_{1}$ or $\beta_{2}$, the stiffer the structure becomes. This increased structure stiffness follows from the fact that the thicker the ligament, the more energy is needed for it to curve.

Before concluding, in accordance with the FEA simulations and the derived mechanical properties of the generalized flexing anti-tetrachiral system, it may be noted that the Poisson's ratio is only dependent on the ratio of the ligament lengths and the relative magnitude of the thicknesses and the radius, where the longer and thinner the ligament in the direction of loading, relative to the ligament in the transverse direction, the more negative the Poisson's ratio. Also, in accordance with the FE simulations, the Young's modulus is dependent on all the parameters considered in this study (i.e. length, thickness, and Young's moduli of the ligaments and radius of the circular nodes). Moreover, it is important to note that in the special case when the properties and dimensions of the horizontal ligaments are the same as those of the vertical ligaments, the Poisson's ratio reduces to -1 and the Young's modulus becomes 


$$
E_{i}=\frac{E_{\mathrm{S}} t^{3}}{6 l r^{2}}
$$

in accordance with the model derived by Grima [13] for the regular flexing anti-tetrachiral system.

5 Conclusions This paper has demonstrated the derivation of the on-axis mechanical properties of generic forms of the anti-tetrachiral systems, which deform through flexure of the ligaments through the use of analytical models that were validated through FEA.

The results suggest that the mechanical properties of the flexing anti-tetrachiral system are highly dependent on the geometry and material properties of the constituent materials. In fact, it appears that the Poisson's ratio of the general flexing anti-tetrachirals is dependent on the ratio of the ligament lengths and the thicknesses (relative to the magnitude of the radius). On the other hand, the Young's moduli of these systems are dependent on all the parameters that were considered, i.e. radius of the nodes, length, and thickness of the ligaments and the Young's moduli of their constituent materials. In particular, for loading in the $O x_{2}$ direction, the Young's modulus increases as the ratios $\alpha_{2} / \alpha_{1}, \beta_{2} / \beta_{1}$, and $E_{\mathrm{s} 2} / E_{\mathrm{s} 1}$ increase. The rigidity of the antitetrachiral system can be changed without affecting the Poisson's ratio, by changing the relative stiffness of the ligaments.

Acknowledgements This work has been funded through the European Union 6th Framework Programme STREP project NMP3-CT-2005-013641 (CHISMACOMB). This research has been carried out using computational facilities procured through the European Regional Development Fund, Project ERDF-080 "A Supercomputing Laboratory for the University of Malta". The authors would also like to acknowledge the financial help received by the Malta Council of Research and Technology (MCST) and the University of Malta.

\section{References}

[1] R. S. Lakes, Science 235, 1038 (1987).

[2] K. W. Wojciechowski, Mol. Phys. 61, 1247 (1987).

[3] R. S. Lakes, Science 238, 551 (1987).

[4] K. E. Evans, J. Phys. D, Appl. Phys. 22, 1870 (1989).

[5] K. W. Wojciechowski, A. Alderson, A. Brańka, and K. L. Alderson, Phys. Status Solidi B 242, 497 (2005).

[6] R. Gatt, J. N. Grima, J. W. Narojczyk, and K. W. Wojciechowski, Phys. Status Solidi B 249, 1313 (2012).

[7] K. W. Wojciechowski, Phys. Lett. A 137, 60 (1989).

[8] L. J. Gibson, M. F. Ashby, G. S. Schajer, and C. I. Roberts, Proc. R. Soc. A 382, 25 (1982).

[9] R. S. Lakes, J. Mater. Sci. 26, 2287 (1991).

[10] D. Prall and R. S. Lakes, Int. J. Mech. Sci. 39, 305 (1997).

[11] O. Sigmund, S. Torquato, and I. A. Askay, J. Mater. Res. 13, 1308 (1998).

[12] J. N. Grima, R. Gatt, and P.-S. Farrugia, Phys. Status Solidi B 245, 551 (2008).
[13] J. N. Grima, PhD Thesis, University of Exeter, Exeter, UK (2000).

[14] M. R. Hassan, F. Scarpa, M. Ruzzene, and N. A. Mohammed, Mater. Sci. Eng. A 481, 654 (2008).

[15] S. Jacobs, C. Coconnier, D. DiMaio, F. Scarpa, M. Toso, and J. Martinez, Smart Mater. Struct. 21, 075013 (2012).

[16] H. Abramovitch, M. Burgard, L. Edery-Azulay, K. E. Evans, M. Hoffmeister, W. Miller, F. Scarpa, C. W. Smith, and K. F. Tee, Compos. Sci. Technol. 70, 1072 (2010).

[17] G. Bergamasco and G. Burriesci, European Patent Bulletin EP1803420B1 (2009).

[18] A. Spadoni, M. Ruzzene, and F. Scarpa, Phys. Status Solidi B 242, 695 (2005).

[19] F. Scarpa, S. Blain, T. Lew, D. Perrott, M. Ruzzene, and J. R. Yates, Composites A 38, 280 (2007).

[20] F. Scarpa, Compos. Sci. Technol. 70, 1033 (2010).

[21] G. Cicala, G. Recca, L. Oliveri, Y. Perikleous, F. Scarpa, C. Lira, A. Lorato, D. J. Grube, and G. Ziegmann, Compos. Struct. 94, 3556 (2012).

[22] W. Miller, C. W. Smith, F. Scarpa, and K. E. Evans, Compos. Sci. Technol. 70, 1049 (2010).

[23] A. Alderson, K. L. Alderson, D. Attard, K. E. Evans, R. Gatt, J. N. Grima, W. Miller, N. Ravirala, C. W. Smith, and K. Zied, Compos. Sci. Technol. 70, 1042 (2010).

[24] A. Lorato, P. Innocenti, F. Scarpa, A. Alderson, K. L. Alderson, K. M. Zied, N. Ravirala, W. Miller, C. W. Smith, and K. E. Evans, Compos. Sci. Technol. 70, 1057 (2010).

[25] K. F. Tee, A. Spadoni, F. Scarpa, Y. J. Liu, and J. S. Leng, J. Vib. Acoust. 132, 031007 (2010).

[26] X. N. Liu, G. L. Huang, and G. K. Hu, J. Mech. Phys. Solids 60, (1907). (2012.

[27] A. Spadoni and M. Ruzzene, J. Mech. Phys. Solids 60, 156 (2012).

[28] Y. J. Chen, F. Scarpa, Y. J. Liu, and J. S. Leng, Int. J. Solid Struct. 50, 996 (2013).

[29] L. Rothenburg, A. A. Berlin, and R. J. Bathurst, Nature 354, 470 (1991).

[30] J. N. Grima and K. E. Evans, J. Mater. Sci. Lett. 19, 1563 (2000).

[31] R. Gatt, D. Attard, E. Manicaro, E. Chetcuti, and J. N. Grima, Phys. Status Solidi B 248, 39 (2011).

[32] K. L. Alderson, A. Alderson, and K. E. Evans, J. Strain Anal. 32, 201 (1997).

[33] J. N. Grima, A. Alderson, and K. E. Evans, J. Phys. Soc. Jpn. 74, 1341 (2005).

[34] J. B. Baker, A. G. Douglass, and A. C. Griffin, Abstr. Pap. Am. Chem. Soc. 210, 146 (1995).

[35] R. H. Baughman, J. M. Shacklette, A. A. Zakhidov, and S. Stafstrom, Nature 392, 362 (1998).

[36] K. W. Wojciechowski, J. Phys. A, Math. Gen. 36, 11765 (2003).

[37] V. V. Novikov and K. W. Wojciechowski, Phys. Solid State 41, 1970 (1999).

[38] K. V. Tretiakov and K. W. Wojciechowski, Phys. Status Solidi B 244, 1038 (2007).

[39] A. A. Pozniak, H. Kaminski, P. Kedziora, B. Maruszewski, T. Strek, and K. W. Wojciechowski, Rev. Adv. Mater. Sci. 23, 169 (2010).

[40] J. W. Narojczyk and K. W. Wojciechowski, Phys. Status Solidi B 244, 943 (2007). 
[41] J. N. Grima, R. Gatt, V. Zammit, J. J. Williams, K. E. Evans, A. Alderson, and R. I. Walton, J. Appl. Phys. 101, 86102 (2007).

[42] R. Gatt, V. Zammit, C. Caruana, and J. N. Grima, Phys. Status Solidi B 245, 502 (2008).

[43] J. M. Gere and B. J. Goodno, Mechanics of Materials, seventh edition (Cengage Learning, USA, 2009), p. 1022.
[44] ANSYS $^{\circledR}$ Release 12.0 (http://www.ansys.com/).

[45] A. Bezazi, F. Scarpa, and C. Remillat, Compos. Struct. 71, 356 (2005).

[46] E. A. Avallone and T. Baumeister, III, Marks' Standard Handbook for Mechanical Engineers, ninth edition (McGraw Hill, New York, 1987). 